\title{
Modelos para avaliação genética da produção de leite no dia do controle nas três primeiras lactações
}

\author{
[Models for genetic evaluation of milk yield on the day of control \\ in the three first lactations] \\ J.C. Reis Filho ${ }^{1}$, R.S. Verneque ${ }^{2}$, R.A. Torres ${ }^{3}$, V.M.P. Ribeiro ${ }^{4}$, F.L.B. Toral ${ }^{4 *}$ \\ ${ }^{1}$ Ministério da Agricultura, Pecuária e Abastecimento - Utra-Viçosa/SFA-MG - Viçosa, MG \\ ${ }^{2}$ Empresa Brasileira de Pesquisa Agropecuária - Juiz de Fora, MG \\ ${ }^{3}$ Universidade Federal de Viçosa - Viçosa, MG \\ ${ }^{4}$ Universidade Federal de Minas Gerais - Belo Horizonte, MG
}

\section{RESUMO}

Objetivou-se verificar se a utilização do modelo autorregressivo (MAR) é adequada para obtenção de parâmetros genéticos para produção de leite no dia do controle (PLDC) de bovinos leiteiros da raça Gir. Foram analisados 125.191 registros de produções diárias, nas três primeiras lactações, por meio dos modelos de repetibilidade (MREP) e MAR. No MREP, foi considerado o efeito de ambiente de curto prazo; no MAR, foi considerado, também, o efeito de ambiente de longo prazo. Os modelos foram comparados por meio do $\log$ aritmo da função de máxima verossimilhança $(-2 \log L)$. A herdabilidade estimada pelo MREP foi 0,18; no caso do MAR, as estimativas para primeira, segunda e terceira lactações foram $0,32,0,28$ e 0,26 , respectivamente. A estimativa de autocorrelação dos componentes de variância de longo prazo foi próxima de zero, e as de curto prazo foram de alta magnitude para primeira $(0,79)$, segunda $(0,79)$ e terceira $(0,81)$ lactações. Logo, a influência do ambiente de curto prazo dentro de cada lactação não é a mesma. $O$ valor de $-2 \log L$ mais próximo de zero foi obtido para o MAR $(-294.884,7778)$ em relação ao MREP (329.266,4810). Assim, o MAR é adequado para obtenção de estimativas de parâmetros genéticos para PLDC nas três primeiras lactações de bovinos leiteiros.

Palavras-chave: autorregressão, Gir leiteiro, herdabilidade, parâmetros genéticos, repetibilidade

\begin{abstract}
Aimed to verify if the autoregressive model (MAR) is adequate to obtain genetic parameters for Gyr dairy cattle milk yield on the test day in the three first lactations. Analysis was performed on 125,191 records of daily production of 9,242 cows using repeatability model (MREP) and MAR. On MREP, a long-term environment was considered, on MAR, the short-term environment was also taken into consideration. The models were compared by logarithm of the maximum likelihood function $(-2 \log L)$. The heritability estimated using the MREP model was 0.18, while the heritability estimated by MAR for first, second, and third lactations were $0.32,0.28$ and 0.26, respectively. The autocorrelation estimates of the components of long-term variance were close to zero, and those of the short-term were of high magnitude for first (0.79), second (0.79) and third (0.81) lactations. Therefore, the influence of the short-term environment within each lactation is not the same. The value of $-2 \log L$ closer to zero was obtained for MAR $(-294,884.7778)$ in relation to MREP $(-329,266.4810)$. Thus, MAR is suitable for obtaining genetic parameters estimates for PLDC in the first three lactations of dairy cattle.
\end{abstract}

Keywords: autorregression, dairy Gyr, genetic parameters, heritability, repeatability

\section{INTRODUÇÃO}

Os principais programas de melhoramento genético de bovinos leiteiros no Brasil têm utilizado a produção de leite acumulada em até

Recebido em 6 de fevereiro de 2017

Aceito em 22 de novembro de 2017

*Autor para correspondência (corresponding author)

E-mail: flbtoral@ufmg.br
305 dias para predição do mérito genético dos animais (Costa et al., 2012; Panetto et al., 2016; Silva et al., 2016). Entretanto, a predição dos valores genéticos realizada por meio de informações de produção de leite no dia do controle (PLDC) pode ser mais acurada que a 


\section{Reis Filho et al.}

predição baseada na produção de leite acumulada em até 305 dias (Sawalha et al., 2005a).

A utilização da PLDC possibilita o emprego dos registros de produção antes mesmo do fim da lactação. Dessa maneira, a avaliação genética de touros pode incluir dados provenientes de um maior número de filhas, o que, além de garantir aumento da acurácia das estimativas, aumenta a disponibilidade de animais jovens para a seleção, $\mathrm{e}$, consequentemente, pode levar ao aumento da intensidade de seleção e à redução do intervalo de gerações (Santos et al., 2013).

Características longitudinais como PLDC, obtidas sequencialmente ao longo do tempo, podem ser analisadas por meio de modelos de regressão aleatória (Torshizi et al., 2012; Santos et al., 2013). Entretanto, o uso desses modelos é, na maioria dos casos, restrito aos registros apenas da primeira lactação, pois incorporar mais de uma lactação para avaliação pode acarretar maior parametrização do modelo. Os registros de PLDC também podem ser avaliados por meio de modelos de repetibilidade (MREP) (Melo et al., 2005). Nesse sentido, as produções no dia do controle são avaliadas como medidas repetidas de uma única característica dentro de uma lactação. Além disso, com MREP é possível incorporar os registros de mais de uma lactação, sem que ocorra necessariamente aumento na parametrização do modelo.

Para que o MREP possa ser aplicado às avaliações genéticas de dados longitudinais, são necessárias algumas pressuposições. Assume-se que a correlação genética entre qualquer ordem de lactação é equivalente a 1,0, a variância fenotípica é a mesma para todas as ordens de lactação e a correlação de ambiente permanente para todas as ordens de lactação é a mesma (Mrode, 2014). Uma vez que nem sempre essas pressuposições são verdadeiras, o MREP pode não ser a melhor alternativa para avaliação dos registros produtivos de uma mesma vaca em diferentes lactações (Reis Filho et al., 2015).

Como é improvável que todos os registros de produção estejam igualmente correlacionados, independentemente da ordem como são avaliados, um processo autorregressivo de primeira ordem pode ser utilizado para modelar estruturas de covariâncias residuais quando os animais possuem medidas repetidas (Quaas,
1984). Nesse sentido, os modelos autorregressivos (MAR) são uma alternativa aos modelos de regressão aleatória e ao MREP, pois possibilitam a avaliação de um maior número de lactações sem aumento do número de parâmetros estimados, além de considerarem diferentes correlações genéticas, ambientais e fenotípicas entre as diferentes lactações.

Nesse contexto, objetivou-se verificar se a utilização do modelo autorregressivo é adequada para obtenção de parâmetros genéticos para produção de leite no dia do controle de bovinos leiteiros da raça Gir.

\section{MATERIAL E MÉTODOS}

Os dados fenotípicos foram provenientes do Programa Nacional de Melhoramento do Gir Leiteiro (PNMGL), obtidos entre 1983 e 2009. $\mathrm{Na}$ preparação do arquivo de dados, somente os controles leiteiros entre o quinto e o $305^{\circ}$ dia das três primeiras lactações de vacas Gir foram utilizados. Os intervalos de idade considerados válidos para primeiro, segundo e terceiro partos foram 24 a 66 meses, 37 a 84 meses e 49 a 102 meses, respectivamente. Os registros de produção leiteira fora do intervalo obtido pela média do dia em lactação $\pm 3,5$ desvios-padrão foram excluídos. As lactações foram classificadas em três níveis de manejo: extensivo (produção a pasto), semiextensivo (produção a pasto com suplementação concentrada) e intensivo (animais confinados e/ ou com uso de substâncias galactogênicas). As produções de leite no dia do controle foram agrupadas em 31 classes de dias em lactação (dentro de cada ordem de parto), com a primeira classe compreendendo as produções aferidas entre o quinto e o nono dia, a segunda do $10^{\circ}$ ao $19^{\circ}$ dia, e assim sucessivamente, com intervalos de 10 dias, com a última classe contendo somente as produções aferidas entre o $300^{\circ}$ e o $305^{\circ}$ dia de lactação. Cada vaca apresentou pelo menos três controles por lactação, sendo a média de 8,3 controles/vaca/lactação.

O arquivo de dados foi composto por 125.191 registros de produções diárias, de 9.242 vacas, em 15.065 lactações. No arquivo final, 4.239, 561 e 328 vacas possuíam registros apenas na primeira, segunda e terceira lactações, respectivamente. Outras vacas tinham duas lactações controladas, sendo $1.771,151$ e 483 
vacas com controles na primeira e segunda, primeira e terceira e segunda e terceira lactações, respectivamente. Somente 1.709 vacas possuíam controles leiteiros nas três lactações. Os números de controles leiteiros realizados nas primeiras, segundas e terceiras lactações foram 64.981, 37.869 e 22.341, respectivamente. Foi utilizado um algoritmo recursivo (Toral e Alencar, 2010) para compor um arquivo de pedigree com 17.483 animais.

Os dados foram analisados por meio de modelos de repetibilidade (MREP) e autorregressivo (MAR). O MREP pode ser descrito da seguinte forma:

$$
\begin{aligned}
y_{\text {hijklmnpq }}=\mu+\operatorname{rap}_{i} & +a c_{j}+m c_{k}+\text { nord }_{m}+\operatorname{man}_{n}+c_{1}\left(\text { icmes }_{r(l)}\right)+c_{2}\left(\text { icmes }_{r(l)}\right)^{2} \\
& +\operatorname{cdel}(\text { op })_{(l)}+a_{h}+p_{h}+t_{q(h l)}+e_{h i j k l m n q}
\end{aligned}
$$

em que: $y_{\text {hijklmnpq }}$ é a produção de leite no dia do controle (PLDC); $\mu$, uma constante geral presente em todas as observações; $r a p_{i}$, o efeito fixo do rebanho-ano do parto $i ; a c_{j}$, o efeito fixo do ano do controle $j ; m c_{k}$, o efeito fixo do mês do controle $k$; nord $_{m}$, o efeito fixo do número de ordenhas diárias $m$; $\operatorname{man}_{n}$, o efeito fixo referente às condições de manejo $n ; c_{1}$ e $c_{2}$ são os coeficientes de regressão linear e quadrático da covariável idade da vaca no controle (em meses); icmes $_{r(l)}$ é a idade da vaca no controle $r$ da ordem de parto $l$ (em meses); $\operatorname{cdel}(o p)_{(l)}$, o efeito da classe de dias em lactação da produção dentro da ordem de parto $l ; a_{h}$, o efeito genético aditivo do animal $h ; p_{h}$, o efeito aleatório de ambiente permanente do animal $h ; t_{q(h l)}$, o efeito aleatório de ambiente de curto prazo, aninhando dentro de vaca e ordem de parto, assumido como independente entre lactações; e $e_{\text {hijklmnpq }}$, o erro aleatório.

Em notação matricial, o modelo de repetibilidade pode ser descrito como: efeitos genéticos aditivos e de ambiente permanente, respectivamente; $a$, o vetor de $y=X \underset{\sim}{\beta}+Z_{\sim} \underset{\sim}{a} Z_{2} \underset{\sim}{p} \underset{\sim}{e}, \quad$ em que: $y$ efeitos genéticos aditivos; $p$, o vetor de efeitos representa o vetor de observações; $X$, a matriz de incidência dos efeitos fixos; $\beta$, o vetor de efeitos fixos; $Z_{1}$ e $Z_{2}$, a matriz de incidência de aleatórios de ambiente permanente; e $e$, o vetor de efeito residual.

Para os efeitos aleatórios, assumiu-se que: $\left[\begin{array}{l}a \\ p \\ e\end{array}\right] \sim N\left(\left[\begin{array}{l}0 \\ 0 \\ 0\end{array}\right],\left[\begin{array}{rrr}G & \phi & \phi \\ & P & \phi \\ \operatorname{sim} & & R\end{array}\right]\right)$ em que: $G=A \sigma_{a}^{2}$, sendo $A$ uma matriz de parentesco genético aditivo entre os animais e $\sigma_{a}^{2}$ a variância genética aditiva; $P=I \sigma_{p}^{2}$, sendo $I$ uma matriz identidade e $\sigma_{p}^{2}$ a variância dos efeitos de ambiente permanente; $R=I \sigma_{e}^{2}$, sendo $I$ uma matriz identidade e $\sigma_{e}^{2}$ a variância residual; e $\phi$ é uma matriz de zeros.

Um modelo de repetibilidade comum é aquele em que os componentes genéticos não aditivos são negligenciáveis e com a pressuposição adicional de uma covariância não genética de medidas repetidas no mesmo animal. A covariância entre resíduos de medidas repetidas no mesmo animal resulta em uma estrutura que pode ser descrita matricialmente nas três medidas repetidas (primeira, segunda, e terceira lactações) como: 


\section{Reis Filho et al.}

$\operatorname{var}\left[\begin{array}{l}e_{1} \\ e_{2} \\ e_{3}\end{array}\right]=\left[\begin{array}{ccc}\sigma_{e}^{2}+\sigma_{p}^{2} & \sigma_{p}^{2} & \sigma_{p}^{2} \\ & \sigma_{e}^{2}+\sigma_{p}^{2} & \sigma_{p}^{2} \\ \operatorname{sim} & & \sigma_{e}^{2}+\sigma_{p}^{2}\end{array}\right]$,

tendo a estrutura de (co)variâncias fenotípicas:

$$
\operatorname{var}\left[\begin{array}{l}
y_{1} \\
y_{2} \\
y_{3}
\end{array}\right]=\left[\begin{array}{ccc}
\sigma_{e}^{2}+\sigma_{p}^{2}+a_{m m} \sigma_{a}^{2} & \sigma_{p}^{2}+a_{m m} \sigma_{a}^{2} & \sigma_{p}^{2}+a_{m m} \sigma_{a}^{2} \\
\operatorname{sim} . & \sigma_{e}^{2}+\sigma_{p}^{2}+a_{m m} \sigma_{a}^{2} & \sigma_{p}^{2}+a_{m m} \sigma_{a}^{2} \\
& & \sigma_{e}^{2}+\sigma_{p}^{2}+a_{m m} \sigma_{a}^{2}
\end{array}\right]
$$

em que: $\sigma_{e}^{2}$ representa a variância única de cada observação; $\sigma_{p}^{2}$, a covariância de ambiente entre pares de medidas de um mesmo animal (com pressuposição de a correlação ser igual em todos os pares); e $a_{m m} \sigma_{a}^{2}$, a covariância genética aditiva sob uma correlação genética unitária entre medidas de um mesmo animal.

É bastante improvável que todos os controles sejam igualmente correlacionados independentemente de sua proximidade. Assim, um modelo mais "realista" foi proposto com a imposição de uma estrutura de covariância autorregressiva para os resíduos (Quaas, 1984).
A mais simples desta estrutura é um processo estacionário autorregressivo de primeira ordem, que é aplicável para intervalos iguais. A estrutura proposta para três medidas repetidas pode ser descrita como:

$\operatorname{var}\left[\begin{array}{l}e_{1} \\ e_{2} \\ e_{3}\end{array}\right]=\left[\begin{array}{ccc}1 & \rho & \rho^{2} \\ & 1 & \rho \\ \operatorname{sim} . & & 1\end{array}\right] \sigma_{e}^{2}=F \sigma_{e}^{2}$, em que: $\sigma_{e}^{2}$ representa a variância ambiental, e $\rho$ representa a autocorrelação com $|\rho|<1$.

Dados contínuos em um modelo autorregressivo são igualmente correlacionados se o intervalo entre eles for constante, o que produz uma correlação decrescente entre dados não contínuos proporcional ao distanciamento no tempo que os separa. A matriz diagonal $F$ de autocorrelação das $n$ amostras pode ser facilmente fatorada em LDL', em que L(L') é uma matriz triangular inferior (superior) e D é uma matriz diagonal. Essa fatorização é especialmente útil para o cálculo do determinante de $F$, por exemplo, para avaliação da função de verossimilhança.
Essa modelagem, contudo, pode criar problemas na interpretação de influências ambientais porque sua estrutura é inteiramente assumida como autocorrelacionada, o que ignora efeitos independentes de um particular dia do controle. Portanto, uma representação potencialmente mais realista para os efeitos aleatórios de ambiente na produção diária de leite, para três observações repetidas, pode ser assim representada:

$\operatorname{var}\left[\begin{array}{l}e_{1} \\ e_{2} \\ e_{3}\end{array}\right]=\operatorname{var}\left[\begin{array}{l}t_{1}+r_{1} \\ t_{2}+r_{2} \\ t_{3}+r_{3}\end{array}\right]=F \sigma_{t}^{2}+I \sigma_{r}^{2}$, em que: $t$ e $r$ são dois componentes de ambiente resultantes de uma partição da variância ambiental $\left(e_{n}\right)$, com $t$ seguindo um processo autorregressivo de primeira ordem em medidas repetidas de produção de leite no dia do controle (PLDC) e $r$ um efeito independente; $\sigma_{t}^{2}$ representa a covariância de ambiente entre medidas repetidas de PLDC; $F$ representa a estrutura de autocorrelação de primeira ordem, que associa as medidas realizadas em cada vaca como definido anteriormente; e $\sigma_{r}^{2}$ representa a variância residual comum a todas as observações. Dessa forma, os dois componentes de ambiente representam influências distintas em cada dado de PLDC. Um componente $\left(\sigma_{t}^{2}\right)$ compreende os efeitos de ambiente de curto prazo, ou seja, aqueles que têm efeito dentro de cada lactação. $\mathrm{O}$ outro componente $\left(\sigma_{r}^{2}\right)$ compreende todas as outras fontes de variação consideradas não temporárias, mas que, de modo independente, afetam a PLDC e, consequentemente, têm efeito entre lactações, como os períodos de serviço anterior e corrente e o período seco anterior (Oliveira et al., 1999; Prosperi et al., 2000). 
O intervalo de tempo desigual entre lactações sucessivas (o período seco) impede de usar o efeito de ambiente de curto prazo para também representar a covariância entre lactações. Em consequência, uma estrutura autorregressiva pode ser uma abordagem realista para representar os efeitos de ambiente de longo prazo, para os quais se abranda a restrição (ou pressuposição) de que as covariâncias entre lactações são iguais e invariantes (Harville, 1979; Quass, 1984). Dessa maneira, o MAR pode ser descrito da seguinte forma:

$$
\begin{gathered}
y_{\text {hijklmnpq }}=\mu+\operatorname{rap}_{i}+a c_{j}+m c_{k}+\operatorname{nord}_{m}+\operatorname{man}_{n}+c_{1}\left(\text { icmes }_{r(l)}\right)+c_{2}\left(\text { icmes }_{r(l)}\right)^{2} \\
+\operatorname{cdel}(\text { op })_{(l)}+a_{h}+p_{h(l)}+t_{q(h l)}+e_{h i j k l m n p q}
\end{gathered},
$$

em que: $p_{h(l)}$ é o efeito aleatório de ambiente de longo prazo, seguindo um processo autorregressivo de primeira ordem entre lactações; $t_{q(h l)}$ é o efeito aleatório de ambiente de curto prazo, aninhando dentro de vaca e ordem de parto, assumido como independente entre lactações, e seguindo um processo autorregressivo de primeira ordem dentro de vaca e entre PLDC, e os demais termos, como definidos anteriormente, para o modelo de repetibilidade, que utilizou a PLDC.

Sob a forma matricial, o modelo autorregressivo pode ser descrito como: $y=X \beta+Z a+M p+Q t+e, \quad$ em que: $\tilde{y} \sim N(X \beta, V) \quad \tilde{\beta}$ representa o vetor desconhecido de efeitos fixos, que, com $X$ conhecido, define a média; $a, p$ e $t$, os vetores de efeitos aleatórios, ambiente permanente de longo prazo e de ambiente de curto prazo, associados com registros em $y$ por $Z, M$ e $Q$, respectivamente; $e$, o vetor de resíduos; e $V$, a matriz de (co)variâncias. As pressuposições de (co)variâncias assumidas para esse modelo são:

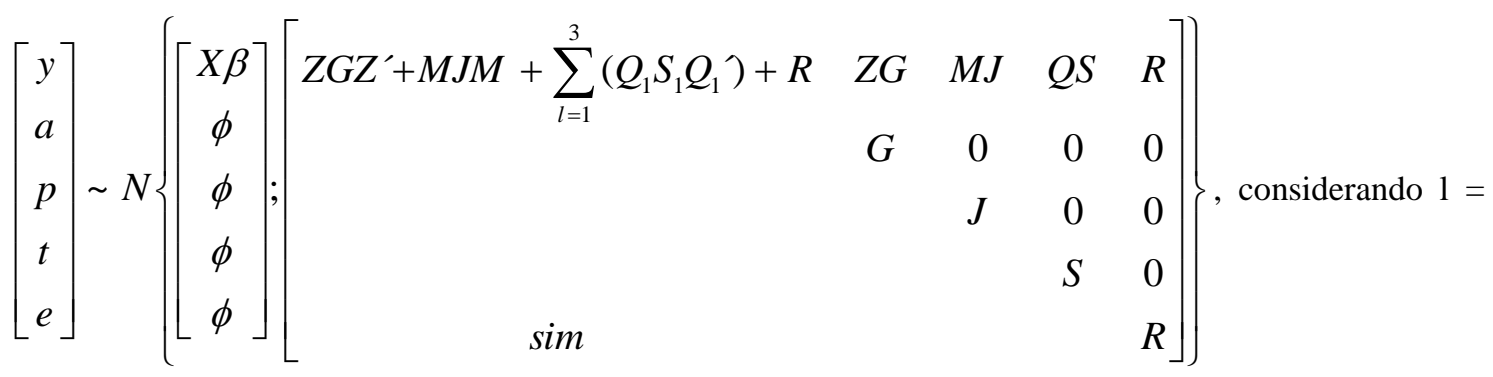
primeira, segunda, e terceira lactações, em que: $G=A \sigma_{a}^{2}, \quad J=\sigma_{p}^{2}\left[\begin{array}{ccc}1 & \rho_{p} & \rho_{p}^{2} \\ & 1 & \rho_{p} \\ \operatorname{sim} & & 1\end{array}\right] \otimes I q_{2}$, 


$$
\begin{aligned}
& S=\left[\begin{array}{lll}
S_{1} & \phi & \phi \\
& S_{2} & \phi \\
\operatorname{sim} & & S_{3}
\end{array}\right], \\
& \operatorname{diag}(F)_{m(l)}=\operatorname{diag}\left[\begin{array}{ccccc}
\rho_{t 1} & \rho_{t 1}^{2} & \ldots & 1 \rho_{t 1}^{n-2} & 1 \rho_{t 1}^{n-1} \\
1 & \rho_{t 1} & & & 1 \rho_{t 1}^{n-2} \\
& \vdots & & & \vdots \\
& & & \rho_{t 1} & \rho_{t 1}^{2} \\
& & \ddots & 1 & \rho_{t 1} \\
\operatorname{sim} & & & 1
\end{array}\right]_{m(l)} \mathrm{em}, R=I_{N} \sigma_{e}^{2},
\end{aligned}
$$

em que: $A$ representa a matriz de parentesco genético aditivo entre os animais; $\sigma_{a}^{2}$, a variância genética aditiva; $J$, a matriz de covariância do ambiente de longo prazo, seguindo um processo autorregressivo de primeira ordem dentro de vaca e entre PLDC; $\sigma_{p}^{2}$, a variância de efeito permanente de ambiente de longo prazo; $\rho_{p}$, o coeficiente de autocorrelação do ambiente de longo prazo (para uma única análise de lactação $J$ reduzida para $I \sigma_{p}^{2} ; S$, a matriz de covariância do ambiente de curto prazo, seguindo um processo autorregressivo de primeira ordem dentro de lactações, que reflete a suposição de independência deste efeito entre as lactações; $F$, uma matriz diagonal autocorrelata correspondente à vaca $m \operatorname{com} n$ amostras no dia do teste na lactação $l ; \sigma_{t 1}^{2}$, a variância do efeito de ambiente de curto prazo do componente $l ; \rho_{t 1}$, o coeficiente de autocorrelação correspondente (para análise de lactação única); $S$ reduz a $\operatorname{diag}\{F\}_{m \sigma_{t}^{2}} ; R$, a matriz de covariância residual; $\sigma_{e}^{2}$, o componente de variância residual; $q_{1}$, o número de animais avaliados; $q_{2}$, o número de registros; $N$, o número total de registros na análise; $I$, uma matriz identidade; $\otimes$, o produto de Kronecker.

As estimativas dos componentes de (co)variância e dos parâmetros genéticos para o MREP foram obtidas por meio do programa Multiple Trait Derivative-Free Restricted Maximum Likelihood (MTDFREML), descrito por Boldman et al. (1995). O MTDFREML utiliza o método da máxima verossimilhança restrita e algoritmo livre de derivadas para estimação dos componentes de variância, e o método simplex para procura dos valores dos componentes de covariância que minimizam a função $-2 \log L$ ( $L$ é a função de verossimilhança). O critério de convergência adotado exigiu variância dos valores do simplex inferior a $10^{-9}$. O procedimento foi repetido três vezes, utilizandose as estimativas obtidas na análise precedente como valores iniciais. Em seguida, foram estimadas as herdabilidades e preditos os valores genéticos. Para identificar estatisticamente o melhor modelo entre o MREP e o MAR, foi utilizado, como critério de ajuste, o logaritmo da função de máxima verossimilhança $(-2 \log L)$.

Para obter as estimativas dos componentes de variância e a predição dos méritos genéticos do modelo autorregressivo, foram utilizadas rotinas do MATLAB (Carvalheira et al., 2002). Na estimação dos componentes de variância, considerou-se que a convergência foi atingida quando a variância do $-2 \log L$ das funções de verossimilhança em todos os pontos definidos no pico do simplex foi $<10^{-9}$, desde que o resultado seja consistente, partindo de diferentes informações de valores iniciais. 


\section{RESULTADOS E DISCUSSÃO}

O modelo autorregressivo (MAR) apresentou melhor ajuste em relação ao modelo de repetibilidade (MREP) para produção de leite no dia do controle nas três primeiras lactações (Tab. 1), uma vez que, para o MAR, foi observado valor de $-2 \log L$ mais próximo de zero.

A variância genética aditiva para produção de leite no dia do controle obtida por meio do modelo autorregressivo foi de maior magnitude quando comparada ao mesmo parâmetro obtido por meio do modelo de repetibilidade (Tab. 1). Esses resultados corroboram aqueles verificados por Carvalheira et al. (1998), para bovinos da raça Holandesa, em que as variâncias genéticas aditivas foram sempre superiores quando estimadas por meio do modelo que considerou o processo autorregressivo de primeira ordem para ambientes de curto prazo sobre a produção de leite em múltiplas lactações, independentemente do ambiente de manejo proporcionado aos animais.

Tabela 1. Estimativas de variâncias ${ }^{1}$ e herdabilidades $\left(h^{2}\right)$ da produção de leite no dia do controle por meio dos modelos de repetibilidade (MREP) e autorregressivo (MAR)

\begin{tabular}{cccccccc} 
& $-2 \log L$ & $\sigma_{a}^{2}$ & $\sigma_{p}^{2}$ & $\sigma_{t}^{2}$ & $\sigma_{e}^{2}$ & $\sigma_{f}^{2}$ & $h^{2}$ \\
\hline MREP & $-329.266,4810$ & 1,72 & 1,89 & 2,19 & 3,83 & 9,64 & 0,18 \\
MAR $^{2}$ & $-294.884,7778$ & 3,00 & $\approx 0$ & & & & \\
MAR OP=1 & & & & 5,78 & 0,60 & 9,37 & 0,32 \\
MAR OP=2 & & & & 7,16 & 0,64 & 10,80 & 0,28 \\
MAR OP=3 & & & & 7,86 & 0,79 & 11,64 & 0,26 \\
\hline
\end{tabular}

${ }^{1}$ Variâncias: genéticas aditivas $\left(\sigma_{a}^{2}\right)$, de ambiente permanente de longo prazo $\left(\sigma_{p}^{2}\right)$, de ambiente de curto prazo $\left(\sigma_{t}^{2}\right)$, residuais $\left(\sigma_{e}^{2}\right)$ e fenotípicas $\left(\sigma_{f}^{2}\right)$. Variâncias expressas em $(\mathrm{kg} / \mathrm{dia})^{2}$. ${ }^{2}$ No MAR, as variâncias $\sigma_{e}^{2}, \sigma_{f}^{2}$ e $h^{2}$ são específicas para cada ordem de parto $(\mathrm{OP}=1,2$ e 3$)$.

Embora a variância de ambiente permanente de longo prazo, estimada por meio do MAR, seja próxima de zero (Tab. 1), os componentes estimados para ambiente de curto prazo (dentro das lactações) foram relativamente elevados. Carvalheira et al. (2002), aplicaram o MAR a dados simulados, em que as PLDC foram consideradas como variáveis longitudinais, seguindo processos autorregressivos de primeira ordem dentro e entre lactações. Os autores concluíram que a análise com uma estrutura de covariâncias autorregressivas de primeira ordem foi eficaz na detecção da presença ou ausência de efeitos autocorrelacionados de ambiente permanente, de longo e curto prazos. Nesse contexto, uma vez que a baixa variância de ambiente permanente de longo prazo não está associada a erros provenientes da modelagem aplicada, esse resultado pode estar associado à estrutura dos dados, que não possuem todas as três lactações para todas as vacas.

As herdabilidades obtidas para PLDC por meio do MAR foram de maior magnitude quando comparadas à herdabilidade obtida por meio do MREP (Tab. 1), e, assim como verificado por Carvalheira et al. (1998), os valores de herdabilidade reduziram de acordo com o aumento da ordem de parto, quando esta foi estimada por meio do MAR. Sawalha et al. (2005b) ressaltam que a estrutura de covariância autorregressiva pode auxiliar na obtenção de estimativas de herdabilidades mais precisas, uma vez que ajusta os efeitos de ambiente permanente ou residuais da PLDC para modelar a covariância entre os efeitos ambientais de maneira mais adequada que o MREP.

As variâncias residuais obtidas por meio do MAR foram de menor magnitude quando comparadas à variância residual obtida por meio do MREP (Tab. 1). Esses resultados corroboram os obtidos por Sawalha et al. (2005b), em que as variâncias residuais para PLDC na primeira ordem de parto, obtidas por meio dos modelos de repetibilidade e autorregressivo foram 12,62 e $7,03 \mathrm{~kg} / \mathrm{dia}^{2}$, respectivamente. É importante ressaltar que a redução na magnitude das 
variâncias residuais verificadas no MAR pode estar associada ao fato de as estruturas autorregressivas permitirem um ajuste flexível das correlações de ambiente, como verificado na Tab. 2.

As estimativas de autocorrelação dos componentes de variância do ambiente de longo prazo foram próximas de zero, e as de curto prazo por ordem de parto foram, de modo geral, de alta magnitude, mas inferiores a 1 (Tab. 2). Esses resultados corroboram os obtidos por Vasconcelos et al. (2004) e demonstram a dificuldade em atender as pressuposições impostas para utilização do modelo de repetibilidade (Mrode, 2014). É possível afirmar, por meio dos resultados obtidos no presente trabalho, que, apesar dos médios a altos valores de correlação entre os ambientes de curto prazo, a influência deste dentro de cada lactação não é a mesma. Nesse sentido, é pertinente a utilização de modelos como o MAR, que levam em consideração a diferença entre os ambientes de curto prazo na avaliação de dados de PLDC em múltiplas lactações.
Tabela 2. Estimativas das autocorrelações dos componentes de variância do ambiente de longo prazo $\left(\hat{\rho}_{p}\right)$ e de curto prazo por ordem de parto $\left(\hat{\rho}_{t l}\right)$, no modelo autorregressivo

\begin{tabular}{cc}
\hline Parâmetro & Estimativa \\
\hline$\hat{\rho}_{p}$ & $\approx 0,00$ \\
$\hat{\rho}_{t 1}$ & 0,79 \\
$\hat{\rho}_{t 2}$ & 0,79 \\
$\hat{\rho}_{t 3}$ & 0,81 \\
\hline
\end{tabular}

As médias das estimativas dos valores genéticos, obtidas por meio do MAR, foram de maior magnitude quando comparadas ao MREP (Tab. 3). Os maiores valores de acurácia foram obtidos quando os valores genéticos foram estimados por meio do MAR, conferindo melhor adequação deste modelo em relação ao MREP. Esses resultados corroboram aqueles obtidos por Sawalha et al. (2005a), em que as acurácias das estimativas por meio do MAR para os grupos de touros $(0,67)$ e vacas $(0,53)$ foram superiores às obtidas por meio do MREP para touros $(0,65) \mathrm{e}$ vacas $(0,51)$.

Tabela 3. Média, desvio-padrão (DP), mínimo e máximo do valor genético e acurácia por meio dos modelos de repetibilidade (MREP) e autorregressivo (MAR)

\begin{tabular}{ccccccc}
\hline & \multicolumn{4}{c}{ Valor genético $(\mathrm{kg})$} & \multicolumn{2}{c}{ Acurácia $(\%)$} \\
\cline { 2 - 7 } Modelos & Média & DP & Mínimo & Máximo & Média & DP \\
\hline MREP & 172,15 & 294,07 & $-724,07$ & $1.426,79$ & 51,99 & 19,99 \\
MAR & 185,01 & 373,54 & $-191,64$ & $2.150,40$ & 60,83 & 22,52 \\
\hline
\end{tabular}

O incremento da acurácia, quando as estimativas são realizadas por meio do MAR, pode estar relacionado ao ajuste das correlações entre os ambientes de curto prazo e de longo prazo (Quaas, 1984), que contribuem para redução da magnitude da variância dos resíduos e consequente aumento da acurácia da predição. Além disso, o MAR proporcionou a maior concentração de animais nas classes com maior acurácia, independentemente do grupo analisado (Fig. 1).

As correlações entre os valores genéticos estimados por meio de ambos os modelos, MREP e MAR, foram de alta magnitude (Tab. 4), sendo as magnitudes das correlações amostrais e de ordem iguais em três grupos de animais.
As menores magnitudes de correlação entre os dois modelos foram verificadas nos grupos de touros 10 e $20 \%$ melhores. É de fundamental relevância atentar para a redução da correlação, em especial nesses grupos, pois é especificamente neles que a utilização de uma metodologia mais adequada poderia acarretar melhores respostas à seleção, uma vez que os melhores touros serão utilizados com maior intensidade. Nesse sentido, deve-se levar em consideração o modelo de melhor ajuste que proporcione menores estimativas de variância residual, maiores estimativas de variância genética aditiva e herdabilidades, bem como maiores acurácias, que, no caso do presente trabalho, foram proporcionadas pelo modelo autorregressivo. 


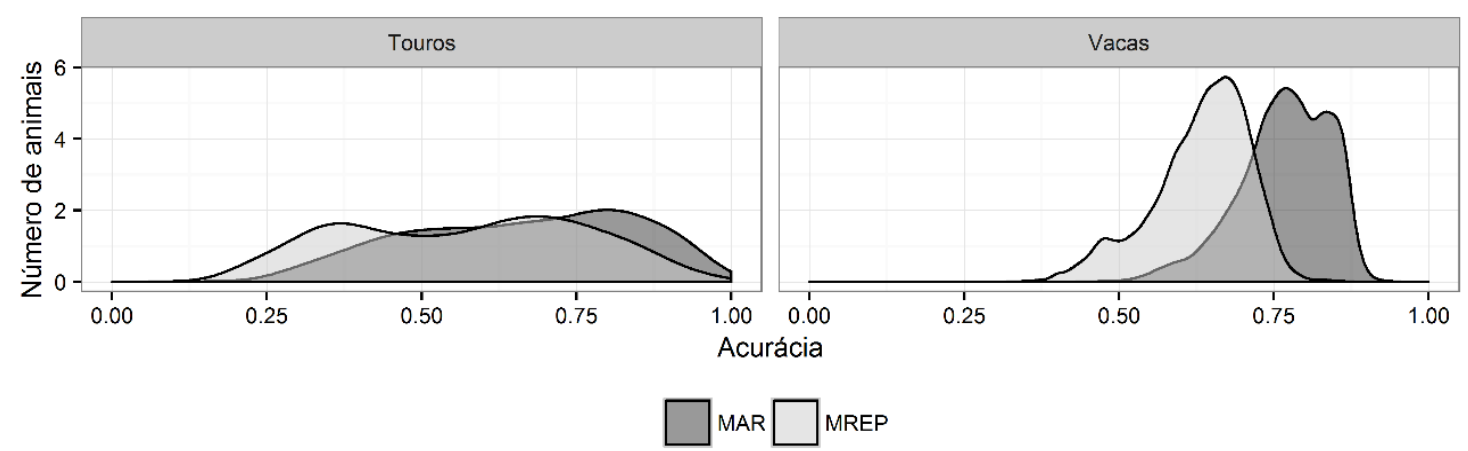

Figura 1. Distribuições das acurácias dos valores genéticos de 817 touros e 9.242 vacas com informações no dia do controle, por meio dos modelos de repetibilidade (MREP) e autorregressivo (MAR).

Tabela 4. Correlações amostrais (Pearson) e de ordem (Spearman) entre os valores genéticos, preditos por meio dos modelos de repetibilidade e autorregressivo

\begin{tabular}{ccc} 
Grupos & Pearson & Spearman \\
\hline Todos os animais do pedigree (17.283 animais) & 0,97 & 0,97 \\
Vacas com produção $(9.242$ vacas) & 0,96 & 0,96 \\
Touros (817 touros) & 0,98 & 0,98 \\
Touros 10\% melhores (82 touros) & 0,90 & 0,83 \\
Touros 20\% melhores (164 touros) & 0,90 & 0,84 \\
\hline
\end{tabular}

\section{CONCLUSÃO}

Com base no logaritmo da função de máxima verossimilhança $(-2 \log L), \quad$ o modelo autorregressivo é adequado para obtenção de estimativas de parâmetros genéticos para produção de leite no dia do controle de bovinos leiteiros e, por isso, constitui uma boa alternativa para a avaliação da produção de leite.

\section{AGRADECIMENTOS}

\section{À FAPEMIG (APQ-01609/16). \\ REFERÊNCIAS}

BOLDMAN, K.G.; KRIESE, L.A.; VAN VLECK, L.D (Eds.). A manual for use of MTDFREML. A set of programs to obtain estimative of variances and covariances [DRAFT]. Beltsville: U.S. Department of Agriculture, Agricultural Research Service, 1995. 125p.

CARVALHEIRA, J.G.V.; BLAKE, R.W.; POLLAK, E.J. et al. Application of an autoregressive process to estimate genetic parameters and breeding values for daily milk yield in a tropical herd of Lucerna and in United States Holstein herds. J. Dairy Sci., v.81, p.27382751, 1998.
CARVALHEIRA， J.G.V.; POLLAK, E.J.; QUAAS, R.L.; BLAKE, R.W. An autoregressive repeatability animal model for test-day records in multiple lactations. J. Dairy Sci., v.85, p.20402045, 2002.

COSTA, C.N.; COBUCI, J.A.; MARQUES, A. et al. (Eds.). Sumário nacional de touros da raça Holandesa. Juiz de Fora: Embrapa Gado de Leite, 2012. 40p.

HARVILLE, D.A. Recursive estimation using mixed linear models with autoregressive random effects. In: VAN VLECK, L.D.; SEARLE, S.R. (Eds.). Proceedings of a conference in honor of C.R. Henderson. Variance components and animal breeding. New York: Cornell University, 1979. p.157-179.

MELO, C.M.R.; PACKER, I.U.; COSTA, C.N.; MACHADO, P.F. Parâmetros genéticos para as produções de leite no dia do controle e da primeira lactação de vacas da raça holandesa. Rev. Bras. Zootec., v.34, p.796-806, 2005.

MRODE, R.A. Linear models for the prediction of animal breeding values. 2.ed. Oxfordshire: CABI Publishing, 2014. 350p. 
OLIVEIRA, A.I.G.; PEREIRA, I.G.; GONÇALVES, T.M.; ARAÚJO, J.V. Período seco anterior e período de serviço sobre a produção de leite e gordura na raça Holandesa no estado de Minas Gerais. Rev. Bras. Zootec., v.28, p.1016-1022, 1999.

PANETTO, J.C.C.; VERNEQUE, R.S.; PEIXOTO, M.G.C.D. et al. (Eds.). Programa nacional de melhoramento do Gir leiteiro sumário brasileiro de touros - resultado do teste de Progênie $-7^{\mathrm{a}}$ prova de pré-seleção de touros maio 2016. Juiz de Fora: Embrapa, 2016. 82p.

PROSPERI, C.P.; OLIVEIRA, A.I.G.; GONÇALVES, T.M. et al. Efeitos do período de serviço corrente e anterior e do período seco sobre a produção de leite de vacas da raça Gir. Ciênc. Agrotec., v.24, p.242-251, 2000.

QUAAS, R.L. Linear prediction. In: QUAAS, R.L.; ANDERSON, R.D.; GILMOUR, A.R. (Eds.). BLUP school handbook. New England: Animal Genetics and Breeding Unit, 1984. p.176.

REIS FILHO, J.C.; VERNEQUE, R.S.; TORRES, R.A. et al. Modelos para avaliação genética da produção de leite em múltiplas lactações. Pesqui. Agropecu. Bras., v.50, p.298$305,2015$.

SANTOS, D.J.A.; PEIXOTO, M.G.C.D.; BORQUIS, R.R.A. et al. Genetic parameters for test-day milk yield, 305-day milk yield, and lactation length in Guzerat cows. Livest. Sci., v.152, p.114-119, 2013.
SAWALHA, R.M.; KEOWN, J.F.; KACHMAN, S.D.; VAN VLECK, L.D. Genetic evaluation of dairy cattle with test-day models with autoregressive covariance structures and with a 305-d model. J. Dairy Sci., v.88, p.3346-3353, $2005 \mathrm{a}$.

SAWALHA, R.M.; KEOWN, J.F.; KACHMAN, S.D.; VAN VLECK, L.D. Evaluation of autoregressive covariance structures for test-day records of Holstein cows: estimates of parameters. J. Dairy Sci., v.88, p.3632-3642, $2005 b$.

SILVA, M.V.G.B.; MARTINS, M.F.; PAIVA, L.C. et al. Programa de melhoramento genético da raça Girolando - sumário de touros resultado do teste de progênie $-4^{\mathrm{a}}$ prova de préseleção de touros - julho 2015. Juiz de Fora, Embrapa, 2016. 72p.

TORAL, F.L.B.; ALENCAR, M.M. Alternatives for analysis of performance data and ranking of Charolais $\mathrm{x}$ Nellore crossbred bulls in performance tests. Rev. Bras. Zootec., v.39, p.1483-1490, 2010.

TORSHIZI, E.T.E.M.; ASLAMENEJAD, A.; NASSIR, M. et al. evaluation of test day milk yield in iranian primiparous. J. Anim. Sci. Adv., v.8, p.664-677, 2012.

VASCONCELOS, J.; MARTINS, A.; PETIMBATISTA, M.F. et al. Prediction of daily and lactation yields of milk, fat, and protein using an autoregressive repeatability test day model. $J$. Dairy Sci., v.87, p.2591-2498, 2004. 